\title{
Modelos Teóricos de Rehabilitación \\ en Salud y la Práctica Fonoaudiológica Una mirada desde los componentes de la CIF
}

\section{Theoretical models of rehabilitation in health and phono-audiological practice: A look from the components of the CIF}

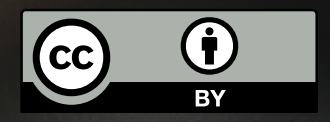

Gloria Esperanza Daza Timana Amparo López Higuera Magda Gissel Mosquera Ruiz Jessica Mildred Erazo Ortiz Enith Adriana Pino Diaz

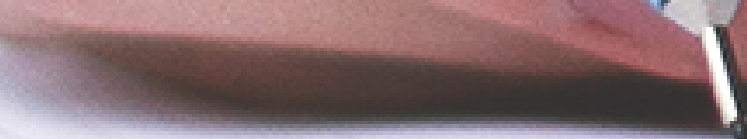
Yersi Estefani Barreto Fernández Morales Rivera
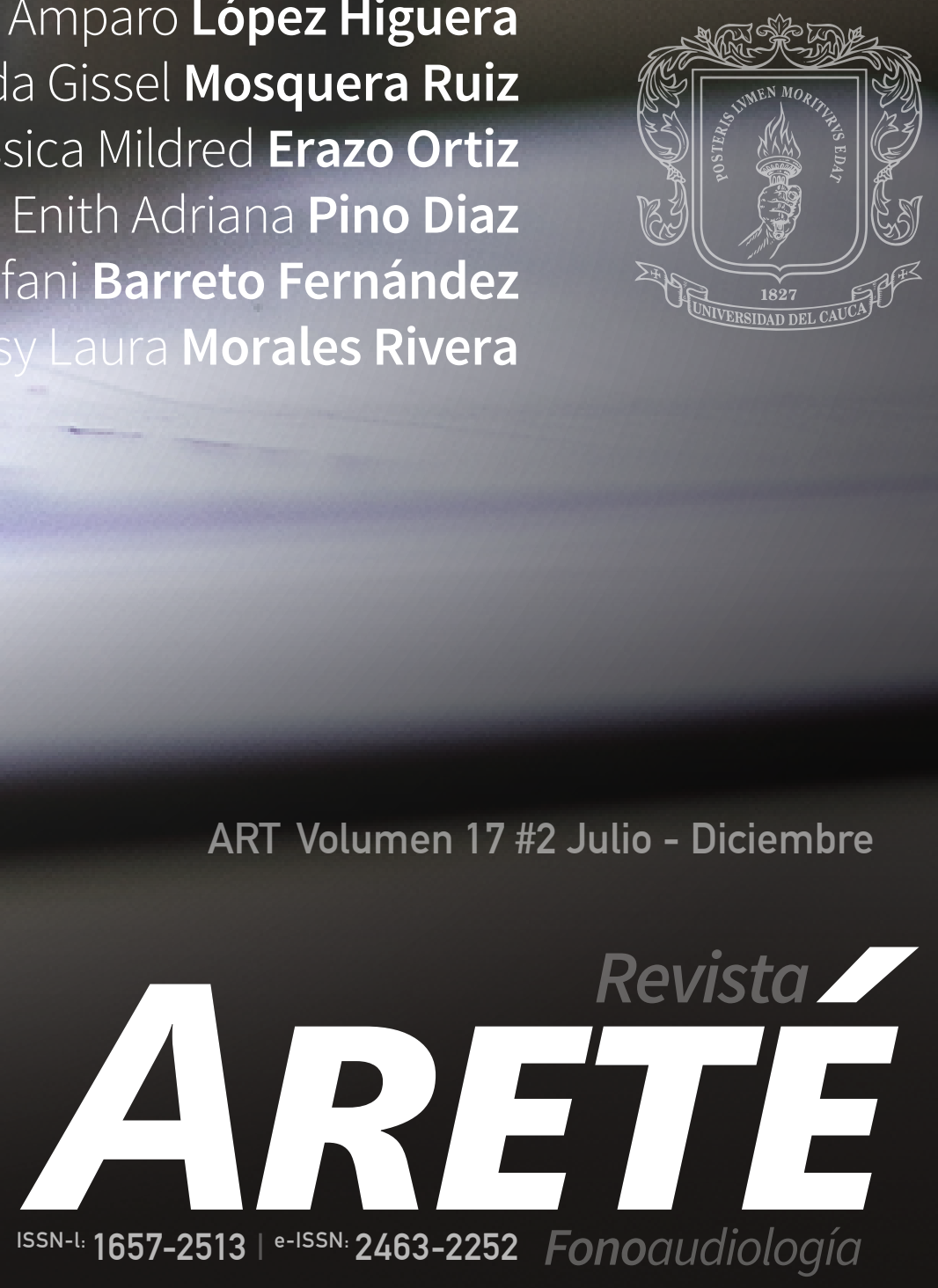
ARETE

$1657-2513$

ID:

1657-2513.art.17204

Title: $\quad$ Theoretical models of rehabilitation in health and phono-audiological practice

Subtitle: A look from the components of the cif

Título: $\quad$ Modelos Teóricos de

Rehabilitación en Salud y la

Práctica Fonoaudiológica

Subtítulo: Una mirada desde los

componentes de la CIF

Alt Title / Título alternativo:

[en]: $\quad$ Models and theories of rehabilitation and clinics of the patologist speech-language and hearing: ICF components

[es]: Modelos teóricos de rehabilitación en salud y la práctica fonoaudiológica: una mirada desde los componentes de la CIF

Author (s) / Autor (es):

Daza Timana, López Higuera, Mosquera Ruiz, Erazo Ortiz, Pino Diaz, Barreto Fernández, \& Morales Rivera

Keywords / Palabras Clave:

[en]: $\quad$ speech; language and hearing sciences; rehabilitation; international classification of functioning; disability and health

[es]: fonoaudiología; rehabilitación; clasificación internacional del funcionamiento; de la discapacidad y de la salud

Submited: 2016-10-20

Acepted: 2017-05-30

\section{Resumen}

El Programa de Fonoaudiología de la Universidad del Cauca, realizó la revisión del modelo de intervención que desde las prácticas de formación se asume, considerando los componentes

de la Clasificación Internacional del Funcionamiento, de la Discapacidad y de la Salud. Se realizó una investigación que contemplo el uso de recursos cuantitativos y cualitativos para realizar el análisis de

80 planeaciones de estudiantes de las prácticas integral I y II. Se concluyó que, aunque el Programa de Fonoaudiología,

ha estado influenciado por el modelo médico biológico, parece estar en estado de transición hacia modelos que contemplan la participación integrada del sujeto, sus necesidades psicológicas y del ambiente.

\section{Abstract}

In the Speech-therapy program at the University of Cauca, the concept of intervention applied in clinical practice during the studies underwent a revision taking into account the components of the International Classification of Functioning, Disability, and Health(ICF). the study using a mixed quantitative and qualitative approach was carried out analyzing 80 treatment plans written by the students for their clinical practices I and II. It was concluded that the speech-therapy program, although it, has been influenced by the biological medical model, seems to be doing a shift towards models traditional include the individuals their psychological needs and their environment.
Gloria Esperanza Daza Timana, ма внs sp

ORCID:

0000-0003-4591-4810

Source | Filiacion:

Universidad del Cauca

BIO:

Fonoaudióloga, Magister en Discapacidad Profesora titular del programa de Fonoaudiología, Universidad del Cauca.

Docente - investigadora vinculada al grupo comunicación Humana y sus Desordenes.

Popayán, Colombia.

City | Ciudad:

Popayan [co]

e-mail:

gdaza@unicauca.edu.co

Amparo López Higuera, MHs вHs sp

ORCID: $\quad$ 0000-0002-7840-3104

Source | Filiacion:

Universidad del Cauca

BIO:

Fonoaudióloga, Magister en Desarrollo

Infantil, Especialista en Pedagogía Infantil. Profesora del programa de

Fonoaudiología, Universidad del Cauca.

Docente - investigadora vinculada al grupo comunicación Humana y sus Desordenes Popayán, Colombia.

City | Ciudad:

Popayan [co]

e-mail:

alopezh@unicauca.edu.co

Magda Gissel Mosquera Ruiz, [BHs]

ORCID: $\quad$ 0000-0001-8779-6395

Source | Filiacion:

Universidad del Cauca

BIO:

Fonoaudióloga, Magister en Discapacidad, Diplomada en Docencia Universitaria.

Profesora del programa de Fonoaudiología, Universidad del Cauca. Docente investigadora vinculada al grupo comunicación Humana y sus Desordenes Popayán, Colombia.

City | Ciudad:

Popayan [co]

e-mail:

magdamosquera@unicauca.edu.co
Jessica Mildred Erazo Ortiz, ORCID: 0000-0002-5054-1089

Source | Filiacion:

Universidad del Cauca

$\mathrm{BIO}$ :

Fonoaudiologo en Formación

City | Ciudad:

Popayan [co]
Enith Adriana Pino Diaz, ${ }^{\text {[внs] }}$ ORCID: 0000-0002-0854-5713

Source | Filiacion:

Universidad del Cauca

BIO:

Fonoaudiologo en Formación

City | Ciudad:

Popayan [co]
Yersi Estefani Barreto

Fernández, $[\mathrm{BH} \mathrm{H}]$

ORCID: 0000-0002-3635-2734

Source | Filiacion:

Universidad del Cauca

BIO:

Fonoaudiologo en Formación

City | Ciudad:

Popayan [co]
Sussy Laura Morales Rivera, ${ }^{\mathrm{BH}}$

ORCID: 0000-0002-0927-5737

Source | Filiacion:

Universidad del Cauca

BIO:

Fonoaudiologo en Formación

City | Ciudad:

Popayan [co]

Citar como:

Daza Timana, G. E., López Higuera, A., Mosquera Ruiz, M. G., Erazo Ortiz, J. M., Pino Diaz, E. A., Barreto Fernández, Y. E., \& Morales Rivera, S. L. (2017). Modelos Teóricos de Rehabilitación en Salud y la Práctica Fonoaudiológica: Una mirada desde los componentes de la cif. Areté issn-l:1657-2513, 17 (2), [pgln]-[pgOut]. Obtenido de: https://revistas.iberoamericana. edu.co/index.php/arete/article/view/1261 


\section{Modelos Teóricos de Rehabilitación en Salud y la Práctica Fonoaudiológica Una mirada desde los componentes de la CIF Theoretical models of rehabilitation in health and phono-audiological practice: A look from the components of the CIF

\author{
Gloria Esperanza Daza Timana \\ Amparo López Higuera \\ Magda Gissel Mosquera Ruiz
} \\ Jessica Mildred Erazo Ortiz \\ Enith Adriana Pino Diaz \\ Yersi Estefani Barreto Fernández \\ Sussy Laura Morales Rivera}

Los modelos de rehabilitación en salud, han evolucionado conforme a los cambios en la comprensión de los conceptos de salud y enfermedad. Así, "se ha pasado de entender los estados de la salud de los individuos desde concepciones mágico religiosas, pasando por lo social y llegando en los últimos tiempos, a comprensiones desde lo biopsicosocial y lo ecológico social” (Guerrero \& León, 2008). A su vez, estos modelos de rehabilitación han influenciado las actuaciones en política sanitaria y social desarrollados en distintos países, inclusive en el ejercicio de la medicina y en general en la atención en salud, dado que las prácticas que corresponden a ellos, han sido transmitidas en los procesos de formación profesional. (Arce, Banguera, \& Velasco, 2012)

Sin embargo, pocas veces los estudiantes de medicina y de otras disciplinas relacionadas con el área de la salud, se han dado a la tarea de hacer un análisis crítico y reflexivo sobre los diferentes modelos teóricos de las unidades didácticas y programas educativos que cursan, y que en últimas afectan su práctica profesional (Arredondo, 1992). En el caso específico de la Fonoaudiología, su historia en Colombia, revela un origen viciado por la influencia del modelo médico de la rehabilitación, lo que supuso en un tiempo la negación del carácter científico y profesional de la ocupación y, por ende, de la autonomía del ejercicio, tal y como lo plantea Cuervo Echeverry. (1998)

Pese a ello, la Fonoaudiología se ha permitido evolucionar y se ha enriquecido de distintas áreas del conocimiento; lo que permitió en primer lugar, su independencia como disciplina de carácter científico y profesional y, en segundo lugar, el paso a modelos diferentes al biomédico, no sólo en la formación de Fonoaudiólogos sino por supuesto en las prácticas que estos llevan a cabo. (Arce, Banguera, \& Velasco, 2012) 


\section{Modelos Teóricos de Rehabilitación en Salud y la Práctica Fonoaudiológica}

Una mirada desde los componentes de la CIF

Según Cuervo Echeverri (1998, pág. 87), la tarea no ha sido fácil, considerando el origen médico de la profesión y la dependencia inicial de los Fisiatras y Foniatras, pero sí necesaria dado que no reflexionar sobre los modelos y las prácticas que estos fecundan pueden significar para el Fonoaudiólogo "pérdida de terreno disciplinario, profesional y político al tiempo que disminuye sus posibilidades de posicionamiento en la sociedad, en los sistemas de seguridad social y educativo y en el mundo académico". Lo anterior explicaría que se haya hecho difícil su participación en ámbitos de atención a la discapacidad, educación inclusiva y ahora desafíos como el postconflicto.

Estos hechos, se convierten en una fuerte razón para que los programas de Fonoaudiología, como el que oferta la Universidad del Cauca, cuyo interés es la formación de profesionales en el ámbito de la rehabilitación, asuman la tarea de reflexionar sobre los modelos desde los que sustentan sus procesos de enseñanza; tema sobre el que además existe una creciente necesidad de investigar.

La evolución de los modelos teóricos que sirven de base a la rehabilitación, se ha dado junto a los cambios en la comprensión de los conceptos de salud, enfermedad y discapacidad. Estos modelos han sido definitivos entre otras cosas, para describir políticas públicas, así como para la formulación de objetivos de formación de profesionales de las áreas de la salud y la rehabilitación.

Distintas perspectivas teóricas, dan forma a cada modelo y por tanto cada uno de ellos lleva implícito concepciones de normalidad, desarrollo e intervención. Para el modelo médicobiológico, por ejemplo, la enfermedad corresponde a desviaciones de la normalidad de la estructura o función de la salud corporal o mental y dado que muchas de ellas no tienen recuperación, solo se puede buscar la adaptación de la persona a las demandas y exigencias de la sociedad. (Padilla-Muñoz, 2010). Esto a su vez, dirigió la intervención hacia la adquisición de algunas habilidades que le permitieran al individuo ser útil económicamente a la sociedad; la ciencia estaba al servicio de ese fin (Albarrán, 2015), lo que se convirtió en un elemento de exclusión.

Como una respuesta al modelo médico, el modelo social planteó que el conjunto de factores que rodean al individuo es el que determina la enfermedad y en algunos casos la discapacidad. (Eroles \& Ferreres, 2002). Este modelo, propone al contexto como la principal fuente de exclusión de las personas con ciertas características (Barton, 1998), y que, en este sentido, la rehabilitación implica no solo la intervención sobre el individuo, sino que las medidas deben ser aplicadas al entorno.

Más contemporáneo, el modelo ecológico de desarrollo humano dio lugar a la elaboración de propuestas de intervención en las que se consideran perspectivas teóricas holísticas frente a la enfermedad y la discapacidad (Albarrán, 2015), así como también a la forma en que las disciplinas científicas y los quehaceres profesionales, ponen al servicio de las personas explicaciones más amplias sobre sus estados de salud.

El modelo bio-psico-social, por su parte integra elementos del modelo médico y del modelo social, razón por la que considera que las condiciones de salud y la discapacidad resultan de la confluencia de factores sociales, necesidades psicológicas y características biológicas individuales (Bernal, Fiqueroa, \& Suárez, 2012; Findlay López, Castaño Franco, Bernal Cano, \& Quintero Uribe, 2014). La influencia de este modelo se ha extendido de manera importante, inclusive la Organización Mundial de la Salud (OMS) lo propone como constructo teórico de la Clasificación Internacional del
Funcionamiento (CIF), el cual paso de ser una clasificación de consecuencias de enfermedades en una relación unidireccional entre las deficiencias y las limitaciones, a una clasificación en la que el funcionamiento de un individuo en un dominio específico se entiende como una relación compleja o interacción entre la condición de salud y los factores contextuales. (Organizacion Mundial de la Salud, 2001)

Además, la CIF comprende una organización en componentes en el que el primero hace referencia al funcionamiento y la discapacidad con respecto a funciones y estructuras corporales, por un lado, y actividad y participación, por otro. En el segundo componente se describen los factores ambientales y personales que regulan las situaciones sociales y los problemas que el individuo experimenta en esas situaciones. (Organizacion Mundial de la Salud, 2001)

Al aplicar la CIF en contextos de rehabilitación, se puede entender la utilidad de evaluar el diferencial existente entre el funcionamiento real y su potencialidad, y permite identificar facilitadores y /o barreras que deben ser objeto de intervención y proporcionar una visión coherente de las diferentes dimensiones de la salud desde una perspectiva biológica, individual y social. (Organizacion Mundial de la Salud, 2001). En este sentido, la aplicación de los componentes de la $\mathbf{C I F}$ con fines de intervención terapéutica es fundamental, en cuanto permite formular un plan de intervención a partir del funcionamiento de la persona y sus necesidades; no solo en relación a las deficiencias, sino también en relación a la limitación en la actividad y/o restricción en la participación social.

Profesiones como la Fonoaudiología encuentran una correspondencia entre sus áreas de desempeño: lenguaje, habla y audición (Ley 376 de 1997), y los componentes de la CIF. Esto a través de funciones y estructuras corporales que están estrechamente relacionadas con la disciplina como:

$\rightarrow$ La función mental especifica del lenguaje

$\rightarrow$ Las funciones sensoriales y dolor

$\rightarrow$ Las Funciones auditivas y vestibulares

$\rightarrow$ Las Funciones de la voz y el habla

Así mismo, hay una relación con el componente que incluye las actividades y la participación, que describe la ejecución individual de tareas y dificultades que tiene una persona en el desenvolvimiento de las situaciones sociales. En este componente aparece un único listado que cubre todo el rango de áreas vitales desde el aprendizaje básico hasta otras áreas más complejas tales como las interacciones interpersonales. Aquí se incluye el aprendizaje y aplicación del conocimiento y la comunicación recepción. (Organizacion Mundial de la Salud, 2001)

Esta relación entre Fonoaudiología y CIF, refiere una necesidad de reflexionar sobre si los procesos de intervención tal y como ha sucedido con la profesión, también han avanzado hacia la apropiación de modelos biopsicosociales abandonando el modelo médico puro. En este sentido, surge el interés de reconocer el modelo de rehabilitación en salud presente en las prácticas de los estudiantes de Fonoaudiología de la Universidad del Cauca y que subyacen a la atención fonoaudiológica.

\section{Materiales y Métodos}

En la investigación se usó el método cuantitativo y cualitativo para acceder a la información de los documentos fuente del estudio. 
En un primer momento la recolección se centró en la recolección de datos de orden cuantitativo (Hernández Sampieri, Fernández Collado, \& Baptista, 2000), lo que facilitó la descripción del contenido de las planeaciones a través de frecuencias considerando las variables: edad, sexo, diagnóstico médico y diagnóstico comunicativo de los usuarios a quienes iban dirigidas, así como áreas, logros y actividades propuestas en función de los constructos de la CIF.

En un segundo momento, la metodología siguió una propuesta de análisis documental con el fin de comprender el sentido del contenido de los textos (López Nogueiro, 2002), en este caso las categorías surgen del establecimiento de relaciones entre la acción del Fonoaudiólogo y los componentes de la CIF.

El corpus inicial estuvo conformado por $\mathbf{1 6 8}$ planeaciones escritas por estudiantes del Programa de Fonoaudiología de la Universidad del Cauca en el II periodo de 2014, en el marco de las prácticas Integral | y II. Finalmente se eligieron $\mathbf{8 0}$ textos que cumplían con los criterios de inclusión; planeaciones de intervención individual, revisadas por el asesor de práctica y cuyos autores cumplieron el requerimiento ético firmando el consentimiento informado. Se excluyeron las planeaciones de evaluaciones y de sesiones de seguimiento y control, así como aquellas que no tenían al menos una revisión por parte del asesor de práctica.

En primer lugar, la revisión documental demandó la selección, registro y sistematización de los documentos en una rejilla construida a partir de las contribuciones de la revisión conceptual, sometida previamente a juicio de expertos y a un ejercicio de aplicación con $\mathbf{1 0}$ textos para precisar su utilidad de acuerdo a los objetivos del estudio. En segundo lugar, se realizó una tarea de codificación de cada uno de los documentos, con el fin de transcribir los apartados que dieran de las categorías de estudio. Este análisis se realizó línea a línea, usando un código para la identificación de cada texto así: P70MAR12 en el que P (Planeación) $\mathbf{7 0}$ (Numero de la planeación) MAR12 (fecha de la revisión).

\section{Resultados}

Es importante indicar que inicialmente se realizó una descripción cuantitativa documental general que dio cuenta de: número de planeaciones y, población a la que se dirigieron, descripción de categorías diagnósticas y áreas de desempeño fonoaudiológico que comprendieron. Luego se realizó un análisis cualitativo de la correspondencia entre logros, actividades y materiales usados, y la noción de deficiencias en estructura, función y participación y categorías relacionadas con los referentes conceptuales.

Los hallazgos mostraron que las planeaciones estaban dirigidas más frecuente a usuarios de $\mathbf{1}$ a $\mathbf{5}$ años con el $\mathbf{2 6 . 3 \%}$, seguido del rango de $\mathbf{6}$ a $\mathbf{1 0}$ años y más de $\mathbf{6 0}$ años cada uno con $\mathbf{1 8 . 8 \%}$. El $\mathbf{6 7 . 5 \%}$ de los documentos analizados no reportaron el diagnóstico médico de los usuarios, sin embargo, entre los diagnósticos que sí se encuentran son frecuentes el "retraso mental, hipoacusia neurosensorial y síndrome convulsivo". Por su parte, el diagnóstico comunicativo con CIE 10 más frecuente fue el trastorno específico del desarrollo del habla y del lenguaje con un $\mathbf{2 2 . 5 \%}$, seguido del trastorno específico de las habilidades escolares con el $\mathbf{1 2 . 5 \%}$ y disfagia con $\mathbf{1 1 . 3 \%}$. Adicionalmente, se encontró que en su mayoría las planeaciones no registraron diagnósticos comunicativos descriptivos (67.5\%).
Sobre las áreas de competencia fonoaudiológica, se encontró que el $\mathbf{5 1 . 1 \%}$ se orientó a la intervención del lenguaje oral, seguido de un $\mathbf{1 6 . 7 \%}$ al lenguaje lecto-escrito. En el área de habla el $\mathbf{1 6 . 1 \%}$ se orientó a la función muscular, seguido de articulación con un $\mathbf{4 . 7 \%}$. Áreas afines como la psicomotricidad y la sensopercepción también fueron objeto de la intervención.

Una parte importante del análisis fue la correspondencia entre las planeaciones y la CIF. La Tabla 1 permite ver con respecto al material reportado en las planeaciones y las funciones corporales, que el $\mathbf{1 6 . 7 \%}$ se relaciona con las funciones mentales, seguido del $\mathbf{1 2 . 9 \%}$ de las funciones de la voz y el habla y el $\mathbf{1 0 . 4 \%}$ con las funciones sensoriales y dolor. Además, se encontró en la correspondencia entre material, y actividad y participación que este se orienta a la comunicación con un $\mathbf{2 3 . 7 \%}$, tareas y demandas generales con un $\mathbf{2 2 . 4 \%}$ y un $\mathbf{8 . 8 \%}$ para aprendizaje y aplicación de conocimiento.

En las planeaciones se encontró que materiales como láminas de vocabulario de categorías, sopa de letras, colores, marcadores, textos, imágenes de dragones, de princesas, títeres, ensartado, animales en semi-concreto, entre otros, fueron usados principalmente en intervenciones dirigidas a población infantil. Por otro lado, materiales como baja lenguas, espejo, gasas, consistencias alimenticias, guantes, jeringa, láminas de categorías semánticas (aseo personal, partes de la casa, partes del cuerpo, alimentos), accesorios personales, calendario móvil, láminas de $A B C$, entre otros, se nominaron en planeaciones dirigidas a adultos. Cabe resaltar que pocos materiales correspondían a objetos de uso cotidiano, por el contrario, se encontró que gran mayoría de los objetos descritos en las planeaciones eran de uso clínico.

Tabla 1 Análisis de la correspondencia entre materiales y los componentes de la CIF

\begin{tabular}{|c|c|c|}
\hline Variable & n: 317 & \\
\hline \multicolumn{3}{|l|}{ Funciones corporales } \\
\hline Funciones mentales & 53 & 16.7 \\
\hline Funciones sensoriales y dolor & 33 & 10.4 \\
\hline Funciones de la voz y del habla & 41 & 12.9 \\
\hline Funciones sistema digestivo metabólico y endocrino & 6 & 1.9 \\
\hline $\begin{array}{r}\text { Funciones neuromusculoesqueléticas y relacionadas con } \\
\text { movimiento }\end{array}$ & 4 & 1.3 \\
\hline \multicolumn{3}{|l|}{ Estructuras corporales } \\
\hline \multicolumn{3}{|l|}{ No corresponde } \\
\hline Aprendizaje y aplicación de conocimiento & 28 & 8.8 \\
\hline Tareas y demandas generales & 71 & 22.4 \\
\hline Comunicación & 75 & 23.7 \\
\hline Movilidad & 5 & 1.6 \\
\hline \multicolumn{3}{|c|}{ Fuente: Elaboración propia } \\
\hline
\end{tabular}

Respecto a los logros y los componentes de la CIF, la Tabla 2, muestra la correspondencia entre logros y funciones corporales, estructuras corporales y actividades y participación. La relación entre logros y funciones corporales se encontró que el $\mathbf{2 7 . 4 \%}$ de los logros están relacionados al trabajo de las funciones mentales dentro de las intervenciones fonoaudiológicas, seguido de funciones de la voz y del habla con un $\mathbf{1 3 . 6 \%}$ y un $\mathbf{6 . 3 \%}$ con las funciones neuromusculoesqueléticas y relacionadas con el movimiento. 
Modelos Teóricos de Rehabilitación en Salud y la Práctica Fonoaudiológica

Una mirada desde los componentes de la CIF

Respecto al logro y las estructuras corporales, se observó que solo el $\mathbf{2 . 2}$ \% de los documentos analizados, se sitúan en el trabajo de las funciones neuromusculoesqueléticas relacionadas con el movimiento, seguido de un $\mathbf{0 . 6 \%}$ con las funciones del sistema digestivo, metabólico y endocrino. Finalmente, en la correspondencia entre logro-actividad y participación se evidenció que el $\mathbf{1 8 . 6 \%}$ de los logros están dirigidos a la comunicación, seguido de tareas y demandas generales con un $12.9 \%$ y aprendizaje y aplicación de conocimiento con un $\mathbf{1 0 . 4 \%}$.

Tabla 2 Correspondencia entre Logros y los componentes de la CIF: Funciones corporales, Estructuras corporales, Actividades y Participación

\begin{tabular}{cccc|}
\hline $\begin{array}{c}\text { Variable } \\
\text { Logro - funciones corporales }\end{array}$ & n: 317 & $\%$ \\
Funciones mentales & 87 & 27.4 \\
$\begin{array}{c}\text { Funciones sensoriales y dolor } \\
\text { Funciones de la voz y del habla }\end{array}$ & 11 & 3.5 \\
$\begin{array}{c}\text { Funciones sistema digestivo metabólico y endocrino } \\
\text { Funciones neuromusculoesqueléticas y relacionadas con } \\
\text { movimiento }\end{array}$ & 3 & 20 & 0.9 \\
$\begin{array}{c}\text { Logro- estructuras corporales } \\
\text { Estructuras relacionadas con los sistemas digestivo. } \\
\text { metabólico y endocrino }\end{array}$ & 2 & 0.6 \\
Estructuras relacionadas con el movimiento & 7 & 2.2 \\
Logro- actividad y participación & & \\
Aprendizaje y aplicación de conocimiento & 33 & 10.4 \\
Tareas y demandas generales & 41 & 12.9 \\
Comunicación & 59 & 18.6 \\
Movilidad & 10 & 3.2 \\
Fuente: Elaboración propia \\
\hline
\end{tabular}

La Tabla 3, relaciona la correspondencia entre actividades, funciones corporales, estructuras corporales, actividad y participación, de esta manera con respecto a las actividades y funciones corporales se observó que el $\mathbf{2 1 . 1 \%}$ estaban orientadas a las funciones mentales, seguido del $\mathbf{1 2} \%$ con las funciones de la voz y del habla, el $\mathbf{1 0 . 1} \%$ con las funciones sensoriales y dolor, y el $\mathbf{0 . 9 \%}$ con las funciones de los sistemas digestivos, metabólicos y endocrinos. En cuanto a la correspondencia entre las actividades planteadas y estructuras corporales se observó que el $\mathbf{0 . 9 \%}$ estaban dirigidas a las funciones neuromusculoesqueléticas, y el $\mathbf{0 . 6 \%}$ a las funciones sensoriales y dolor.

Así mismo, en actividades y participación se evidenció que el $\mathbf{2 2 . 7 \%}$ de las actividades estaban dirigidas a la comunicación, el $\mathbf{1 4 . 2} \%$ en tareas y demandas generales y el $\mathbf{1 1} \%$ en aprendizaje y aplicación de conocimiento.

Tabla 3 Correspondencia entre actividades y funciones corporales, estructuras corporales, actividad y participación

\begin{tabular}{|ccc}
\hline $\begin{array}{c}\text { Variable } \\
\text { Logro - funciones corporales }\end{array}$ & n: 317 & $\%$ \\
$\begin{array}{c}\text { Funciones mentales } \\
\text { Funciones sensoriales y dolor }\end{array}$ & 67 & 21.1 \\
Funciones de la voz y del habla & 38 & 10.1 \\
Funciones sistema digestivo metabólico y endocrino & 3 & 0.9
\end{tabular}

$\begin{array}{llll}\text { Funciones neuromusculoesqueléticas y relacionadas con } & 17 & 5.4\end{array}$

Logro- estructuras corporales

Estructuras relacionadas con los sistemas digestivo. metabólico y endocrino

Por su parte, el análisis cualitativo se presentó de acuerdo a las subcategorías que emergieron después de revisar las planeaciones bajo la consigna de buscar relaciones entre la acción del Fonoaudiólogo y los componentes de la CIF - Tabla 4.

Tabla 4 Categoría de Análisis: Componentes de la CIF y acción del Fonoaudiólogo.

\begin{tabular}{|c|c|c|c|}
\hline & Componente CIF & Área de & $\begin{array}{l}\text { Subcategorías de análisis } \\
\text { Interés de la intervención }\end{array}$ \\
\hline \multirow{8}{*}{$\begin{array}{l}\text { 오 } \\
\text { 옹 }\end{array}$} & \multirow{4}{*}{$\begin{array}{l}\text { Función y } \\
\text { estructura }\end{array}$} & \multirow[b]{2}{*}{ Lenguaje } & $\begin{array}{l}\text { Interés por el desarrollo de las } \\
\text { funciones del lenguaje. }\end{array}$ \\
\hline & & & $\begin{array}{c}\text { Interés por el desarrollo de funciones } \\
\text { de codificación y decodificación de } \\
\text { mensajes. }\end{array}$ \\
\hline & & \multirow[b]{2}{*}{ Habla } & Interés por la producción oral. \\
\hline & & & $\begin{array}{c}\text { Interés por las relaciones sensoriales y } \\
\text { neuromusculoesqueléticas. }\end{array}$ \\
\hline & $\begin{array}{l}\text { Actividad y } \\
\text { participación }\end{array}$ & Lenguaje & $\begin{array}{l}\text { Aprendizaje y uso del conocimiento y la } \\
\text { comunicación. }\end{array}$ \\
\hline & $\begin{array}{l}\text { Función y } \\
\text { estructura }\end{array}$ & $\begin{array}{l}\text { Lenguaje y } \\
\text { habla }\end{array}$ & $\begin{array}{l}\text { Aplicabilidad del uso del lenguaje oral, } \\
\text { lecto-escrito y la producción del habla. }\end{array}$ \\
\hline & \multirow[t]{2}{*}{$\begin{array}{c}\text { Actividad y } \\
\text { participación }\end{array}$} & $\begin{array}{c}\text { Lenguaje y } \\
\text { habla }\end{array}$ & $\begin{array}{l}\text { Aplicabilidad de los aprendizajes y de la } \\
\text { competencia comunicativa }\end{array}$ \\
\hline & & & Fuente: Elaboración propia \\
\hline
\end{tabular}

\section{Análisis frente a los logros}

El interés por el desarrollo de las funciones del lenguaje y el desarrollo de funciones de codificación y decodificación de mensajes, es una categoría que emerge de la búsqueda de la correspondencia entre logros, la función y la estructura. Se identificó que los logros mostraban un interés particular por el desarrollo de las funciones mentales que incluyen el lenguaje y la codificación y decodificación de mensajes. Los siguientes logros están dirigidos al área de lenguaje que se incluye dentro de las funciones mentales de acuerdo a la conceptualización CIF. Los verbos del logro hacen énfasis en la necesidad de los usuarios de dar uso a las funciones del lenguaje.

p1nov20 "Utilizar las funciones del lenguaje como: interaccional, personal, informativa de forma correcta"

p2nov20 "Usar la función imaginativa de forma correcta y de manera autónoma"

p69nov20 "Usar la función imaginativa del lenguaje de forma correcta y autónoma en sus intercambios comunicativos"

La comprensión de mensajes a través del lenguaje lectoescrito, también encuentra relación con las funciones mentales, esto dado que la CIF afirma que estas se relacionan con el reconocimiento y la utilización de signos, símbolos y otros componentes del lenguaje. Algunos de los ejemplos de logros dirigidos a esta función son:
p18nov20
"Comprensión de textos narrativos tipo textos cortos"
p63nov20 "Comprender textos instructivos de forma literal, inferencial y crítica"
p57nov20 "Comprender textos narrativos tipo cuento" 
Igual que la primera, la categoría del interés por el desarrollo de las funciones del habla: la producción oral y su relación con funciones y estructuras sensoriales y neuromusculoesqueléticas, surge de la búsqueda de la correspondencia entre logros, la función y la estructura. Específicamente sobre las funciones de la voz y el habla, se encontraron logros en relación a la producción articulatoria, la alimentación y otras funciones y estructuras relacionadas. La articulación, por ejemplo, está implicada en la producción de sonidos y del habla según la CIF; razón por la que logros dirigidos a la articulación están asociados a la función de la voz y el habla.

p2nov20 "Articular el fonema /g/ en sílaba directa en posición inicial, media de palabra, de forma correcta de manera autónoma"

p12nov20 "Articular palabras bisílabas por imitación que contengan los fonemas $/ \mathrm{m} / \mathrm{y} / \mathrm{p} /{ }^{\prime \prime}$

p34nov19 "Articular por imitación todas las vocales"

p58nov20 "Articular correctamente y por demanda el sinfón /dr/ en posición inicial, media y final de palabra"

Generalmente, los logros enfocados a la articulación estaban acompañados de otros que según la $\mathbf{C I F}$ corresponden a funciones de la voz y el habla no especificadas, pero que contribuyen a la producción oral.

p1 nov20 "Coordinar movimientos labiales, linguales y mandibulares de forma aproximada por imitación"

p19nov20 "Coordinar praxias activas labiales, linguales, faciales, y de soplo de manera correcta con velocidad y fuerza correcta"

p20nov20 "Coordinar praxias activas labiales y linguales con adecuada fuerza y velocidad por imitación de manera correcta"

En línea con lo anterior, también se encontraron logros que comprometían la acción sensorial; funciones sensoriales y de dolor que abarca todas las funciones de los sentidos, vista, oído, tacto, gusto, etc., así como de la sensación de dolor y temperatura. Algunos ejemplos son:

p39nov20 "Responder ante estímulos táctiles y propioceptivos con contracciones de la musculatura exo y endobucal".

p51nov20 "Responder con contracciones y movimientos de O.F.A ante la estimulación táctil."

p8nov20 "Reaccionar a estímulos olfativos y tácticos utilizando objetos en concreto"

Otro proceso del habla que surge continuamente dentro de los logros, es la alimentación. Esta puede ubicarse en las funciones del sistema digestivo metabólico y endocrino, dado que la CIF indica que la deglución junto con la ingesta y eliminación hacen parte de dichas funciones. A continuación, se citan algunos ejemplos:

p14nov20 "Desencadenar el reflejo deglutorio con fluidos propios y líquido espeso tipo néctar"

p46marzo11"Mantener un selle labial para evitar el derramamiento de alimentos durante la fase oral de la deglución"

Por su parte, algunos logros ya no se dirigieron directamente a las funciones y estructuras corporales, sino más bien a la actividad y participación; particularmente en el área de lenguaje. Los logros dirigidos a la lectura, por ejemplo, se incluyeron en aprendizaje y aplicación de conocimiento, dado que este componente incluye aprendizajes básicos como: copiar, repetir, aprender a leer, aprender a escribir, aprender a calcular, entre otras.

\section{p30nov18 "Leer textos descriptivos con un tipo lector corriente con apoyo" \\ p70marzo12"Leer un texto narrativo tipo cuento "el gran partido" de forma silábica vacilante sin apoyo de forma oral, con comprensión a nivel literal e inferencial sin apoyo".}

Otra de las actividades relacionadas con el aprendizaje y la aplicación de conocimiento a las que se dirigieron los logros, fue a la comprensión de órdenes y la construcción de oraciones, lo que, de acuerdo a la CIF, incluye aspectos generales y específicos de la comunicación a través del lenguaje, los signos o los símbolos, incluyendo la recepción y producción de mensajes, llevar a cabo conversaciones y utilización de instrumentos y técnicas de comunicación.

\section{p4nov20 "Ejecutar órdenes simples con apoyo" \\ p19nov20 "Ejecutar órdenes simples con objeto presente de manera correcta" \\ p3nov20 "Construir oraciones subordinadas adversativas con la estructura: art+sust+verb+compl unidas con la conjunción 'pero' de forma correcta por demanda" \\ p10nov20 "Crear oraciones complejas a partir de un texto sin apoyo"}

\section{Análisis de las actividades}

Como en los logros, en la revisión de las actividades se identificaron intereses frente a las funciones, más que a las estructuras. Las descripciones de actividades y las consignas que las acompañan muestran una estrecha relación entre el logro, la actividad y como se entrenan acciones de la vida diaria. De esta relación surge la categoría aplicabilidad del uso del lenguaje oral y lecto-escrito y la correspondencia entre actividades, funciones y estructuras.

Las actividades dirigidas a las funciones mentales del lenguaje; relacionadas con el reconocimiento y la utilización de signos, símbolos y otros componentes del lenguaje, así como las funciones de recepción y decodificación del lenguaje oral, pueden observarse a modo de ejemplo a continuación:

p63nov2

"Dar inicio a la escritura de un texto informativo, tarjeta para la tía. Enseñar algunas frases que puede utilizar para la elaboración de las tarjetas. Tratar de que utilice todos los elementos gramaticales que dan coherencia y cohesión a un texto, así como también que haga uso adecuado de los signos de puntuación, acentuación y exclamación, corregir los errores de escritura que presente [...].

"Bueno, inicia escribiendo la tarjeta a tu tía, primero escribe lo que le quieres decir en la tarjeta puedes ayudarte con el siguiente texto, escúchame lo voy a leer. Aquí nos dan algunas ideas aquí hay algunas frases con las que puedes 
Modelos Teóricos de Rehabilitación en Salud y la Práctica Fonoaudiológica

Una mirada desde los componentes de la CIF

dar inicio o que puedes incluir en lo que vas a escribir, te las voy a leer [...]"

Otras funciones mentales a las cuales estaban dirigidas las actividades son las de orientación; relacionadas con el conocimiento y que permiten establecer relaciones de lugar, tiempo y persona.

p13nov20 Establecimiento de una conversación: "buenos días (nombre del usuario) ¿cómo está?

¿Cuénteme que hizo en la mañana desde el momento en que se levantó?".

"Presentación del calendario móvil para identificación de ayer, hoy y mañana. "(Nombre del usuario) ¿Qué día es hoy? si hoy es lunes, ¿qué día fue ayer, y qué día será mañana?"

Otra categoría que se indujo del análisis, fue el interés de la aplicabilidad de la producción del habla y su correspondencia entre actividades, funciones y estructuras. Se encontraron los ejemplos de las actividades que fueron clasificadas en las funciones de la voz y el habla; como la producción de diferentes sonidos mediante el paso de aire a través de la laringe y con el flujo y ritmo del habla.

p40marzo2 "Ejercicios de tonificación glótica: -Sentado con los brazos sobre las piernas, inspirar y en la pausa que empuje la nuca contra la mano del terapista y diga /cric-cric-cric/, /quic-quicquic/y /cuc-cuc-cuc/-Colocar la mano sobre la frente del paciente y éste apoyará la cabeza ligeramente inclinada haciendo fuerza hacia delante, diciendo guic-guic-guic. -Sentado con brazos relajados, producir una $/ \mathrm{m} /$ prolongada para que dé, el tono adecuado para que las emisiones posteriores sean con el mismo tono: Espirar emitiendo oclusivas posteriores: / $k-g / y$ vocales cerradas $/ i, u, e /$, manteniendo en sonido, alargando sobre la vocal y terminando sobre una oclusiva sorda al final de la sílaba, llevando la entonación hacia el agudo como preguntando: quiiiic? cuuuuc? gueeeec? - Se va aumentando el número de series en cada espiración y se van introduciendo todas las vocales"

Así mismo se encontraron actividades que estuvieron encaminadas al trabajo de las funciones de los sistemas digestivo metabólico y endocrino, y funciones neuro-músculo esqueléticas relacionadas con el movimiento.

p15nov20 "Así mismo se dará pastas al paciente para que realice una adecuada deglución durante la ingesta de alimento".

p50marzo11"Iniciar el trabajo de succión variando la forma, tamaño y grosor del pitillo, iniciando con líquidos claros"

p43marzo 11 "Durante toda la terapia realizar control estabilizando la cabeza y la cintura escapular con las manos para que no se desorganice y sostenga durante unos segundos dichas posiciones. Se usarán también recordatorios (tocar el hombro - orden verbal) para que la paciente se intente organizar de forma autónoma"

También se encontraron actividades dirigidas a las funciones sensoriales y dolor; en las que se intervienen las funciones de los sentidos, vista, oído, gusto, etc., así como de la sensación de dolor. p6nov20 "Asociar un olor a un sabor "bien NN ahora yo le voy a colocar esto en la nariz, ¿listo? mire NN así huele y así sabe (limón, naranja, café)"

p37nov20 "Identificación de figura fondo por medio de estímulo auditivo y estimulo táctil. "doña NN le voy a pintar las uñas" mientras se le harán sonar instrumentos para ver si la paciente reacciona al sonido"

p70mar12 "Discriminación de palabras que contengan el fonema /d/ "muy bien ahora yo te voy a decir dos palabras y tú me vas a decir cuál tiene la /d/ ¿listo?"

Finalmente, se encontró una categoría referida al interés de la aplicabilidad de los aprendizajes y de la competencia comunicativa y su relación con las actividades y participación, por ejemplo, en el ámbito del aprendizaje y aplicación de conocimiento, que según la CIF trabaja sobre el aprendizaje, la aplicación de los conocimientos aprendidos, el pensamiento, la resolución de problemas y la toma de decisiones. Algunos ejemplos de dichas actividades son:

p28nov18 "Realización de la lectura del texto narrativo tipo cuento "la tortuga y la rana" la primera parte del cuento a cargo de la practicante y el final a cargo de la usuaria. "NN vamos a empezar esta sesión leyendo un cuento muy interesante y divertido, voy a empezar a leer y yo te digo cuando sigas $t u^{\prime \prime}$

Además, se encontró que algunas actividades estaban dirigidas a las tareas y demandas generales, las cuales tratan sobre los aspectos generales relacionados con la puesta en práctica de tareas sencillas diarias y la recepción y producción de mensajes, llevar a cabo conversaciones y utilización de instrumentos y técnicas de comunicación.

p5nov20 "realización de ordenes con una puerta "bueno NN mire bien cómo es que se llama esta? bien ahora míreme haga lo que yo le pida abra la puerta cierre la puerta, muy bien abra la puerta y salga, abra la puerta entre y cierre la puerta muy bien NN míreme y lo hace usted"

p7nov2 "Presentación de carteles de norma de cortesía: presentarse y despedirse para que logre identificar en qué momento se las utiliza. Luego se las pegara en un cuadro "NN mira estas laminas ¿qué está haciendo el señor? ¿Cómo usted saluda a las personas? ¿Cómo pedir permiso? ¿cómo se despide a una persona?"

\section{Discusión}

Con respecto a las variables sociodemográficas de estudio, se encontró que las intervenciones estuvieron encaminadas a la atención de usuarios ubicados principalmente en el rango de edad $\mathbf{1}$ a $\mathbf{5}$ años, seguido del rango de los $\mathbf{6}$ a $\mathbf{1 0}$ años. La mayoría de los usuarios se encontraban cursando grados de básica primaria y el diagnóstico comunicativo de mayor frecuencia fue el trastorno especifico del desarrollo del lenguaje y del habla, con el código F80.0 según la Clasificación Internacional de Enfermedades CIE10. (Organizacion Panamericana de la Salud, 2008) 
Además, el área en la que se enfocaron la mayoría de planeaciones fue el lenguaje, lo que podría estar directamente relacionado con la población predominante a la que van dirigidas las intervenciones en las prácticas de Fonoaudiología, es decir la población infantil. Lo anterior guarda relación con los resultados obtenidos en el estudio realizado por Guevara Agredo, Muñoz Zambrano, y Campo Granados (2011), quienes refieren, que la población infantil resulta ser el principal sujeto de atención.

Los diagnósticos encontrados en las planeaciones obedecen a modelos biológicos principalmente, las tablas de frecuencia permitieron ver como prevalecen los diagnósticos médicos que indican causas orgánicas, todos se acompañan del código CIE10, y pocas veces de diagnósticos descriptivos que den cuenta de manera específica y detallada de las deficiencias en lenguaje, habla y audición, y su relación con el comportamiento comunicativo cotidiano.

Las áreas hacia las cuales estuvieron enfocadas las intervenciones fueron en su orden lenguaje y habla seguidas de la sensopercepción, lo que podría explicarse con el hecho de que referentes conceptuales previamente revisados indican que las áreas disciplinares hacia las que se enfoca la investigación y actuación del Fonoaudiólogo son el lenguaje, habla, comunicación y audición, siendo el área de lenguaje a la que más se orienta la investigación y la intervención en Fonoaudiología. (Pava-Ripoll, Payan Villamizar R, \& Reyes Torres, 2011)

Se encontró que la mayoría de los logros en las intervenciones fonoaudiológicas, están encaminados a las funciones corporales, lo que parecería estar en aproximación con el modelo médicobiológico, y en similar número a la actividad y la participación lo que corresponde a modelos sociales y biopsicosociales. Igual sucede en el caso de las actividades, en porcentajes similares se reparten aquellas dirigidas a las funciones corporales y a la actividad y participación.

Estos resultados permiten relacionar prácticas de atención con los modelos de rehabilitación desde los referentes conceptuales aportados por Cespedes (2005) y, Findlay López y Cols. (2014), en este caso parece ser que las prácticas de intervención del Programa de Fonoaudiología están divididas entre el modelo médico, en el que el síntoma está asociado directamente a una parte del cuerpo y lo biopsicosocial que incluye la participación y realización de tareas en contextos diferentes.

La misma dicotomía entre modelos de rehabilitación se encontró en relación a los materiales utilizados para las intervenciones, hay una leve superioridad de aquellos enfocados a la actividad y participación sobre los que están dirigidos a las funciones corporales. Esto parece reafirmar el hecho de que exista una transición del modelo médico-biológico a modelos más integradores, dejando ver que las intervenciones de los estudiantes de Fonoaudiología están en un proceso de trasformación, determinado en cierta forma por las políticas públicas que se han establecido en los últimos años en el país, tendencia que reafirma Seelman. (2004)

Contrario a lo dicho anteriormente sobre el modelo predominante en las intervenciones de los estudiantes de Fonoaudiología, se encuentra lo referido por Hoover y Ortiz (2012), al indicar que en las tendencias epistemológicas el modelo de discapacidad predominante fue el modelo Biopsicosocial, seguido del Modelo Social.

Sobre el análisis documental, se identificó que los logros y actividades planteados para el área del lenguaje tienen correspondencia con el modelo CIF en el componente funciones y estructuras, ya que se identifica al lenguaje como una función mental que permite la comunicación. Estos ejemplos concuerdan, con los resultados reportados por Pava-Ripoll, Payan Villamizar R y Reyes Torres (2011), que indican que desde la atención fonoaudiológica hay interés por la intervención de las funciones mentales.

Sin embargo, la búsqueda de aspectos comunes en las planeaciones permitió ver que los logros reflejan interés para que los usuarios aprendan y usen la comunicación, a esto se agrega que cuando se trata de asociar las actividades del lenguaje a la actividad y la participación, otras dimensiones CIF juegan un papel importante, por ejemplo, aquellas que se relacionan con la realización de tareas y demandas generales usadas en la vida cotidiana. Lo anterior coincide con lo descrito por Fernández López, y otros (2009), quienes especifican que la actividad, se refiere a la ejecución individual de tareas, las cuales están relacionadas con los factores personales y ambientales de cada individuo, en tanto que la participación se refiere al desenvolvimiento en las situaciones sociales y los problemas que el individuo experimenta.

Sobre los logros y actividades relacionadas con el área del habla, estos se orientaron al trabajo de las funciones del sistema digestivo, metabólico, endocrino y a las funciones neuromusculoesqueléticas y las relacionadas con el movimiento. La CIF clasifica dentro del componente de estructuras corporales a las funciones relacionadas con la deglución, la ingesta y la eliminación, así como también las funciones relacionadas con el movimiento y la movilidad, incluyendo las funciones de los huesos, los músculos, las articulaciones y los reflejos, lo que pareciera dar al área del habla intereses propios de procesos clínicos. Al respecto Cruz, Fernández y García (2012), refieren que en algunas áreas las intervenciones se centran en tratamientos e intervenciones clínicas con una clara tendencia al llamado modelo médico de rehabilitación. Sin embargo, la búsqueda de aspectos comunes en las planeaciones también permitió ver que desde el área de habla se busca la aplicabilidad cotidiana de sus procesos, por ejemplo, alimentarse.

Este estudio permitió identificar en las propuestas de intervención individual realizadas por los estudiantes de Fonoaudiología, al menos dos tendencias en la aplicación de modelos de rehabilitación. Por un lado, las propuestas de intervención delimitan las deficiencias de los usuarios a través de diagnósticos no descriptivos lo cual corresponde al modelo médico. Sin embargo, los logros y las actividades mostraban intereses divididos entre las funciones mentales y la actividad y la participación que incluyen subcategorías como el aprendizaje, la aplicación de conocimiento y la comunicación. En línea con esto, la mayoría de los materiales citados en las propuestas, están enfocados a la actividad y la participación y en menor porcentaje a funciones corporales.

También se encontró que, en la intervención de las áreas del lenguaje y el habla, propias del quehacer fonoaudiológico, los logros y las actividades se acompañaron de acciones en favor de procesos cognitivos como la memoria y la orientación, y procesos relacionados con la postura y el movimiento.

A pesar de que la Fonoaudiología, por años ha estado influenciada por el modelo médico biológico, se han llevado a cabo recientes reflexiones que la ubican en un estado de transición hacia modelos que contemplan la participación integrada del 


\section{Modelos Teóricos de Rehabilitación en Salud y la Práctica Fonoaudiológica}

Una mirada desde los componentes de la CIF

sujeto, sus necesidades psicológicas y el ambiente. En tal sentido, para el Programa de Fonoaudiología de la Universidad del Cauca, así como para la profesión, es enriquecedor analizar sus propuestas de intervención desde el modelo conceptual de la CIF. Esto permite encontrar lugar a comprensiones no solo biológicas, sino también sociales de los fenómenos de salud y enfermedad.

\section{Agradecimientos}

Los investigadores agradecen a la Universidad del Cauca, especialmente a la Vicerrectoría de Investigaciones y a la comunidad académica del Programa de Fonoaudiología por su apoyo para realizar esta investigación.

\section{Referencias}

Albarrán, A. (Julio- Diciembre de 2015). Algunas perspectivas y modelos de comprensión de la discapacidad. (P. Garcia Avendaño, Ed.) Revista Venezolana de Análisis de Coyuntura, XXI(2), 127 - 165. Obtenido de http://190.169.94.12/ojs/index.php/rev_ac/article/view/11295/11002

Arce, V., Banguera, K., \& Velasco, Y. (Julio de 2012). Historia y panorama actual de la fonoaudiología en Colombia. Cali: Universidad del Valle. Obtenido de Fundamentos fonoaudiologicos: https:// fonoaudiologiaunivalle.jimdo.com/panorama-actual-en-colombia/

Arredondo, A. (1992). Análisis y reflexión sobre modelos teóricos del proceso salud enfermedad. Cadernos de Saúde Pública, 8(3), 254-261. doi:https://doi.org/10.1590/50102-311X1992000300005.

Barton, L. (1998). Discapacidad y Sociedad. (L. Barton, Ed.) España: Ediciones Morata.

Bernal, P., Fiqueroa, K., \& Suárez, S. (2012). Aportes del paradigma sistémicoecológico a la disciplina fonoaudiológica. Cali: Universidad del Valle. Obtenido de https://fonoaudiologiaunivalle.jimdo.com/paradigmas-enfonoaudiolog\%C3\%ADa/sist\%C3\%A9mico-ecol\%C3\%B3gico/

Cespedes, G. M. (2005). La nueva cultura de la discapacidad y los modelos de rehabilitación. AQUICHÁN. Obtenido de http://aquichan.unisabana. edu.co/index.php/aquichan/article/view/64/733

Cruz, I., Fernandez, A., \& García, S. (2012). Hacia la definición de una agenda estratégica de investigación en Colombia. Conferencia presentada en el Foro Nacional de Discapacidad. Congreso de la República de Colombia.

Cuervo Echeverri, C. (1998). La profesión de fonoaudiología: Colombia en perspectiva internacional. Bogotá D.C., Colombia: Facultad de Medicina - Universidad Nacional de Colombia. Obtenido de http:// www.bdigital.unal.edu.co/2344/2/clemenciacuervoecheverri.7998.pdf
Eroles, C., \& Ferreres, C. (2002). La discapacidad, una cuestion de derechos humanos. Argentina, Buenos Aires: Espacio. Obtenido de http:// www.convenciondiscapacidad.es/Publicaciones_new/4_Libro\%20 Agustina\%20Discapacidad.pdf

Fernandez López, J. A., Fernandez Fidalgo, M., Geoffrey, R., Stucki, G., \& Cieza, A. (2009). Funcionamiento y discapacidad: la clasificación internacional del funcionamiento (CIF). Revista Española de Salud publica

Findlay López, D. M., Castaño Franco, M. A., Bernal Cano, T., \& Quintero Uribe, J. F. (2014). Enfoques de intervención fonoaudiológica en las universidades del suroccidente colombiano. (G. E. Rodríguez Jiménez, Ed.) Areté, 14(1), 65-81. Obtenido de http://revistas.iberoamericana. edu.co/index.php/arete/article/view/709/619

Guerrero, L., \& León, A. (2008). Aproximación al concepto de salud. Revision histórica. Revista Venezolana de Sociología y Antropología, 610-630.

Guevara Agredo, A., Muñoz Zambrano, I., \& Campo Granados, A. (2011). Situación laboral de los egresados del programa de fonoaudiología de la Universidad del Cauca. Areté, 11(1), 152-164. Obtenido de: https:// revistas.iberoamericana.edu.co/index.php/arete/article/view/383

Hernández Sampieri, R., Fernández Collado, C., \& Baptista, L. (2000). Metodología de Investigación. México: Mc Graw Hill.

Hoover Vanegas, J., \& Ortiz, M. (2012). Tendencias investigativas en discapacidad en instituciones de Educación Superior del Suroccidente Colombiano 2000 - 2009. Trabajo de Grado- Maestría en Discapacidad, Manizales. Obtenido de http://repositorio.autonoma. edu.co/jspui/bitstream/17182/301/1/

Ley 376, Por la cual se reglamenta la profesión de Fonoaudiología y se dictan normas (CONGRESO DE COLOMBIA 04 de Julio de 1997). Obtenido de https://www.mineducacion.gov.co/1759/ articles-105005_archivo_pdf.pdf

López Nogueiro, F. (2002). El análisis de contenido como método de investigación. Revista de educación, XXI(4), 172.

Organizacion Mundial de la Salud. (2001). Clasificacion Internacional del Funcionamiento, la Discapacidad y la Salud. Madrid. Obtenido de https://aspace.org/assets/uploads/publicaciones/e74e4-cif_2001.pdf

Organizacion Panamericana de la Salud. (2008). Correspondencia entre actividades y funciones corporales, estructuras corporales, actividad y participación. (CIE-10). USA: Pan American Health Organization.

Padilla-Muñoz, A. (Enero-Junio de 2010). Discapacidad: con texto,concepto y modelos. Revista Colombiana de Derecho Internacional(16), 381-414.

Pava-Ripoll, N. A., Payan Villamizar R, C. M., \& Reyes Torres, A. (Diciembre de 2011). Aportes desde la investigación formativa a la producción en Fonoaudiología: el caso de una universidad colombiana. Revista da Sociedade Brasileira de Fonoaudiologia, 16(4), 377-383. doi:https:// doi.org/10.1590/S7516-80342011000400003

Seelman, C. (2004). Tendencias en la Rehabilitación y en la Discapacidad: Transición desde un Modelo Médico a un Modelo de Integración. Disabiliy Word, 22 